\title{
Influence of the 48867A $>C$ (Asp358Ala) IL6R polymorphism on response to a lifestyle modification intervention in individuals with metabolic syndrome
}

\author{
V.R.A. Vargas ${ }^{1,2,3}$, S.L. Bonatto ${ }^{1}$, F.E. Macagnan ${ }^{4}$, A.M.P. Feoli ${ }^{4}$, \\ C.S. Alho ${ }^{1}$, N.D.V. Santos ${ }^{3}$ and V.M. Schmitt ${ }^{3}$ \\ ${ }^{1}$ Programa de Pós-Graduação em Biologia Celular e Molecular \\ Faculdade de Biociências, Pontifícia Universidade Católica do Rio Grande do Sul, \\ Porto Alegre, RS, Brasil \\ ${ }^{2}$ Universidade Regional Integrada do Alto Uruguai e das Missões, \\ Santo Ângelo, RS, Brasil \\ ${ }^{3}$ Laboratório de Biologia Molecular, Faculdade de Farmácia, \\ Pontifícia Universidade Católica do Rio Grande do Sul, Porto Alegre, RS, Brasil \\ ${ }^{4}$ Faculdade de Enfermagem, Nutrição e Fisioterapia, \\ Pontifícia Universidade Católica do Rio Grande do Sul, Porto Alegre, RS, Brasil \\ Corresponding author: V.M. Schmitt \\ E-mail: vmschmitt@pucrs.br \\ Genet. Mol. Res. 12 (3): 3983-3991 (2013) \\ Received July 16, 2012 \\ Accepted November 10, 2012 \\ Published February 28, 2013 \\ DOI http://dx.doi.org/10.4238/2013.February.28.8
}

\begin{abstract}
We evaluated the response of individuals with metabolic syndrome to lifestyle modification intervention and examined the influence of the 48867A $>$ C (Asp358Ala) IL6R (rs2228145) polymorphism on this response. Participants were randomly divided into two groups: NI, nutritional intervention; NIE, nutritional intervention and exercise practice. Intervention lasted three months and participants completed a comprehensive evaluation and had blood collected for biochemical measurements.
\end{abstract}


Eighty-two sedentary individuals with at least three criteria for metabolic syndrome were included. Comparing metabolic syndrome parameters before and after intervention, a reduction of waist circumference was observed, although significant only for AA and AC genotypes. Also, a decrease in triglyceride levels was observed (significant for AA genotype individuals; for the AC genotype, only in the NIE group). Significant reduction of fasting glucose level was observed in all AA genotype individuals; for the AC genotype, only in the NI group. Systolic blood pressure showed significant reduction in AA and AC genotype individuals. After three months of lifestyle modification intervention, improvement in some of the metabolic syndrome parameters was observed, some associated with the IL6R genotype. At enrollment, participants with genotypes $\mathrm{AA}$ and $\mathrm{AC}$ showed more severe conditions regarding metabolic syndrome inclusion criteria, supporting previous reports that the A allele is a genetic risk factor. These individuals, however, had a better response to intervention compared to individuals with the CC genotype, suggesting that nutritional control and exercise practice could prevent risks associated with metabolic syndrome more efficiently in individuals bearing the A allele.

Key words: Nutritional intervention; Exercise; Obesity; Genetic risk factor

\section{INTRODUCTION}

Metabolic syndrome (MetS) is a condition characterized by a clustering of metabolic risk factors, including abdominal obesity, high blood pressure, hypertriglyceridemia, low levels of high-density lipoprotein cholesterol (HDL-C) and hyperglycemia. The presence of MetS increases the risk for developing cardiovascular disease, diabetes, disability, and mortality (Lakka et al., 2002; Yamada et al., 2007; Cornier et al., 2008; Duvnjak and Duvnjak, 2009; Meshkani and Adeli, 2009; Gupta and Gupta, 2010; Akintunde et al., 2011). MetS development has been attributed to environmental factors (diet $v s$ bad food habits; physical activity $v s$ sedentary lifestyle), as well as to genetic polymorphisms known to be risk factors for cardiovascular disease, diabetes or inflammatory conditions (Lakka et al., 2002; Yamada et al., 2007; Ordovas and Shen, 2008; Meshkani and Adeli, 2009; Dalle et al., 2010; Monteiro and Azevedo, 2010).

Interleukin 6 (IL-6) is a cytokine mediator of the inflammatory process and has been associated with dyslipidemia, type 2 diabetes, and MetS (Fernández-Real and Ricart, 2003; Wolford et al., 2003; Wang et al., 2005). IL-6 binds to its receptor, IL6R, initiating an intracellular signaling cascade that leads to an inflammatory state. IL6R has two subunits: the transmembrane $130-\mathrm{kD}$ subunit (IL-6ST, IL6R $\beta$, or gp130), responsible for signal transduction with consequent activation of the inflammatory state, and the $80-\mathrm{kDa}$ subunit (IL6R $\alpha$ or gp80), to which IL-6 binds (Fernández-Real and Ricart, 2003; Wang et al., 2005). A soluble and biologically active form of IL6R (sIL6R) is present in the plas- 
ma, corresponding to the extracellular portion (gp80), produced by differential splicing or by proteolytic cleavage of IL6R (shedding) (Galicia et al., 2004; Esteve et al., 2006). When IL-6 binds to sIL6R, this complex can bind to gp130 present on the surface of cells that do not have IL-6R (and so would not respond to the inflammatory stimuli of IL-6), leading to the dimerization of gp130, consequent intracellular signaling (trans-signaling), resulting in a diffuse inflammatory state (Jones et al., 2001; Heinrich et al., 2003; Esteve et al., 2006; Qi et al., 2007; Rafiq et al., 2007; Santer et al., 2010).

Seven single nucleotide polymorphisms (SNPs) have been identified so far in the gene coding for IL6R (Kim et al., 2003; Qi et al., 2007; Rafiq et al., 2007; Santer et al., 2010). Among those, the SNP located at exon 9 [48892A $>$ C; Asp358Ala; rs2228145 (rs8192284 merged into rs2228145)] has been associated with elevated serum levels of sIL6R (Galicia et al., 2004). Polymorphisms of the IL6R gene have been implicated in many studies on obesity, insulin resistance, metabolic syndrome, and risk for diabetes, showing a positive relationship of the A allele (Asp) with obesity and/or type 2 diabetes (Wolford et al., 2003; Hamid et al., 2005; Wang et al., 2005; Esteve et al., 2006).

Lifestyle modification based on behavior therapy is known to be the most important and effective strategy to manage metabolic syndrome (Villareal et al., 2006; Yu et al., 2009). For example, weight reduction represents the main goal of most intervention studies on MetS since it is associated with significant improvement in the clinical abnormalities of MetS, including glucose levels, lipid profile, and blood pressure (Villareal et al., 2006; Dalle et al., 2010). However, the relationship between genetic polymorphism in the IL6R gene and metabolic syndrome has not been investigated in the Brazilian population. The purpose of this study was to evaluate the response to a lifestyle modification intervention in individuals with metabolic syndrome and to investigate if polymorphism $48867 \mathrm{~A}>\mathrm{C}$ (rs2228145; Asp358Ala) of the IL6R gene exerts any influence on this response.

\section{MATERIAL AND METHODS}

\section{Subjects}

Study subjects were participants of the MERC study ("Effect of lifestyle modification on cardiovascular risk factors that comprise the diagnostic criteria for metabolic syndrome, inflammatory markers, and autonomic balance"), a multidisciplinary program conducted at Pontifícia Universidade Católica do Rio Grande do Sul (PUCRS), in Rio Grande do Sul State, Brazil.

Inclusion criteria for the MERC study were the presence of three or more MetS criteria (according to I-DBSM; for glucose, the new IDF definition was considered): a) abdominal circumference: $>88 \mathrm{~cm}$ for women and $>102 \mathrm{~cm}$ for men; b) blood pressure: systolic $\geq 130 \mathrm{mmHg}$ and diastolic $\geq 85 \mathrm{mmHg}$; c) fasting plasma glucose level: $\geq 100 \mathrm{mg} /$ dL; d) triglycerides: $\geq 150 \mathrm{mg} / \mathrm{dL}$; e) HDL-C: $<40 \mathrm{mg} / \mathrm{dL}$ for men and $<50 \mathrm{mg} / \mathrm{dL}$ for women (Grundy et al., 2005; Sociedade Brasileira de Cardiologia, 2005; IDF Clinical Guidelines Task Force, 2006). Also, individuals should be sedentary.

All participants underwent a comprehensive evaluation, including age, anthropometric measures (weight, height), blood pressure, and waist circumference (WC). Blood 
samples were collected from participants before $(t=0)$ and after three months of intervention ( $\mathrm{t}=3$ months) for glucose, HDL-C and triglycerides evaluation, determined at Laboratório de Patologia Clínica do Hospital São Lucas (HSL)/PUCRS (Vitros ${ }^{\circledR}$ Chemistry System 5.1 Fusion, Ortho Clinical Diagnostics, Johnson \& Johnson, Rochester, NY, USA).

At enrollment, participants were randomly divided into two groups of three months lifestyle modification intervention: nutritional intervention group (NI), with participants receiving a dietary plan based on recommendations of the I-DBSM at enrollment and a follow-up every two weeks; nutritional intervention and exercise group (NIE), with participants receiving the same nutritional intervention as the NI group with an additional physical activity program consisting of 30-min continuous walking on an ergometric treadmill at an intensity of 60 to $70 \%$ of maximum heart rate, three times per week. Participants of both groups completed the whole three months intervention.

\section{IL6R Asp358Ala polymorphism}

Genomic DNA was extracted from peripheral blood samples collected with $5 \mathrm{~mL}$ EDTA (Lahiri and Nurenberg, 1991). 48867A >C IL6R SNP (rs2228145; Asp358Ala) was assayed according to Esteve et al. (2006). A fragment of $286 \mathrm{bp}$ containing the polymorphic region was amplified by polymerase chain reaction (PCR) with primer sense 5'-AAG GTT CCT TTG AGG CTT TT-3' and antisense 5'-CCA TAA ATT TCA GAA TGG GC-3'. PCR conditions were $10 \mathrm{mM}$ Tris- $\mathrm{HCl}, \mathrm{pH} 8.5,50 \mathrm{mM} \mathrm{KCl}, 2 \mathrm{mM} \mathrm{MgCl}_{2}, 25$ pmol of each primer, $0.4 \mathrm{mM}$ dNTPs, $0.5 \mathrm{U}$ Taq DNA polymerase (EasyTaq ${ }^{\mathrm{TM}}-4 \mathrm{G}$ ), and $1 \mu \mathrm{g}$ extracted DNA. Amplification program was $94^{\circ} \mathrm{C}$ for $5 \mathrm{~min}, 35$ cycles of $94^{\circ} \mathrm{C}$ for $1 \mathrm{~min}$, $57^{\circ} \mathrm{C}$ for $1 \mathrm{~min}$, and $72^{\circ} \mathrm{C}$ for $1 \mathrm{~min}$, and a final extension at $72^{\circ} \mathrm{C}$ for $7 \mathrm{~min}$. PCR products were digested with HinfI restriction enzyme (Invitrogen) and genotypes defined by restriction fragment length polymorphism, with allele $\mathrm{C}$ remaining uncut (290 bp), and allele A generating two fragments (172 and $118 \mathrm{bp}$ ).

\section{Data analysis}

For statistical analysis, the Wilcoxon or Student $t$, Mann-Whitney, and Kruskal-Wallis tests were used when appropriate. Bonferroni's correction was applied for multiple comparisons.

\section{Ethics}

This study was conducted according to the guidelines laid down in the Declaration of Helsinki and all procedures involving human subjects were approved by the Research Ethics Committee of Pontifícia Universidade Católica do Rio Grande do Sul. Written informed consent was obtained from all participants.

\section{RESULTS}

\section{Characteristics of study participants}

A total of 82 participants of the MERC study were included: NI counted 42 par- 
ticipants and NI + NIE 40 participants. The mean age of the participants was 50.89 years (33-61) and females were the majority $(\mathrm{N}=60 ; 73.2 \%)$. Comparing gender and age distribution in the NI and NIE groups, no significant difference was observed $(\mathrm{P}>0.05)$. Also, no statistical difference was observed in any of the MetS inclusion criteria comparing the NI and NIE groups at baseline $(\mathrm{P}>0.05)$. The mean values of the MetS inclusion criteria for the study participants were as follows: WC $104.25( \pm 8.93) \mathrm{cm}$ for women and 110.45 $( \pm 7.68) \mathrm{cm}$ for men; triglycerides $201.94( \pm 106.63) \mathrm{mg} / \mathrm{dL}$; HDL-C $46.82( \pm 9.72) \mathrm{mg} /$ $\mathrm{dL}$ for women and $38.36( \pm 8.64) \mathrm{mg} / \mathrm{dL}$ for men; fasting plasma glucose $105.96( \pm 35.45)$ $\mathrm{mg} / \mathrm{dL}$; systolic blood pressure $131.51( \pm 15.64) \mathrm{mmHg}$; and diastolic blood pressure $82.57 \mathrm{mmHg}( \pm 10.49)$.

\section{IL6R Asp358Ala polymorphism}

48867A $>$ C IL6R polymorphism was determined for 77 subjects, since 5 DNA samples were not properly amplified. Genotype frequencies for participants were AA $36.4 \%(\mathrm{~N}=28)$, AC $46.7 \%(\mathrm{~N}=36)$ and $\mathrm{CC} 16.9 \%(\mathrm{~N}=13)$. Allelic frequency was 0.60 for A and 0.40 for $\mathrm{C}$. In the NI and NIE groups, genotypic distribution was, respectively, AA $33.3 \%(\mathrm{~N}=13)$ and $39.5 \%(\mathrm{~N}=15)$; AC $46.7 \%(\mathrm{~N}=36)$ and $43.6 \%(\mathrm{~N}=17)$; $\mathrm{CC} 23.1 \%(\mathrm{~N}=9)$ and $10.5 \%(\mathrm{~N}=$ 4). Allelic distribution was for the NI group 0.55 for A and 0.45 for C, while for the NIE group it was 0.64 for A and 0.36 for C. All were in Hardy-Weinberg equilibrium (HWE).

\section{$48867 \mathrm{~A}>\mathrm{C}$ genotype and lifestyle modification}

Table 1 presents the comparison of anthropometric and biochemical parameters of the study group (all participants and according to intervention group) regarding genotype and instance of sample collection ( $t=0$, before intervention; $t=3 \mathrm{mo}$, after three months of intervention). The analysis of $\mathrm{WC}$ according to genotypes and groups showed a general reduction, although significant only for AA and AC individuals. Regarding triglyceride levels, a decreasing trend was observed, except for NI AC genotype. A significant reduction was observed only for NIE AA and AC genotypes. No significant changes were observed in HDL-C levels. Significant reduction in fasting plasma glucose level was observed in AA genotype individuals of the NI and NIE groups, but for AC genotype only participants of the NI group had a significant reduction. Systolic blood pressure showed significant reduction in AA and AC genotype individuals in general, but in the intervention groups it was observed only in NI AC individuals. No significant change was observed in diastolic blood pressure.

\section{DISCUSSION}

Data in the literature disclose an association between Asp358Ala IL6R polymorphism and circulating levels of sIL6R, with A allele (Asp) related to higher levels, both in homozygosity and heterozygosity, with a consequent increase in sIL6R/IL-6 complex, leading to a systemic inflammatory condition (Wolford et al., 2003; Galicia et al., 2004; Wang et al., 2005; Esteve et al., 2006). More recently, it has been suggested that obesity can induce a chronic inflammatory state with associated insulin resistance, possibly by inhibiting the insulin receptor signaling pathway (Wellen and Hotamisligil, 2005; Das and Elbein, 2006; De Luca and Olefsky, 2006; Lazar, 2006). 


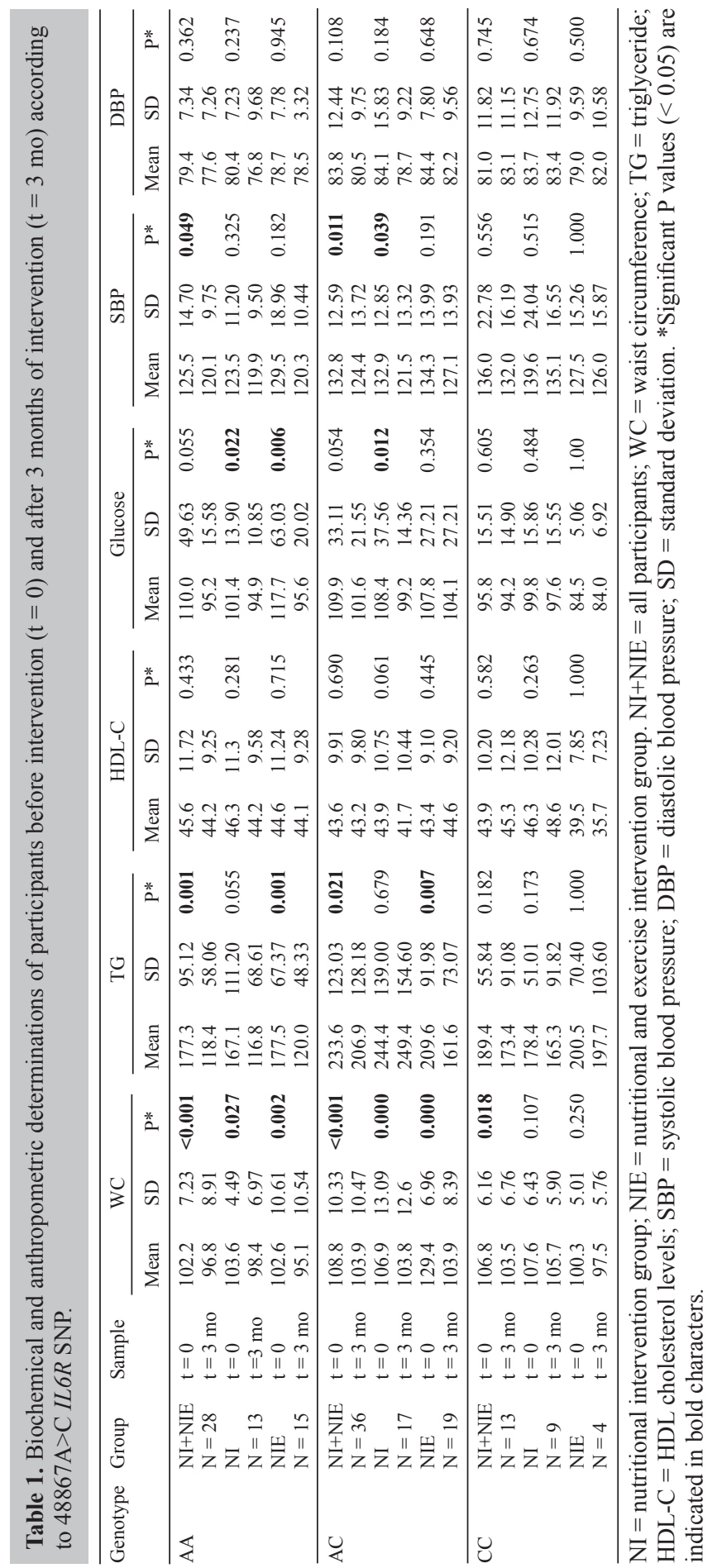


The Asp358Ala IL6R polymorphism frequency found in our study is similar to that reported in previous studies (Galicia et al., 2004; Hamid et al., 2005; Esteve et al., 2006; Qi et al., 2007; Rafiq et al., 2007). Moreover, the A allele frequency found in our study was in accordance with data from dbSNP database (Sherry et al., 2001) prevalence for the Caucasian population ranging from 0.50 to 0.70 . Most of the participants in our study were Caucasians, and the prevalence of the A allele was 0.6, showing a distribution as in worldwide reports.

In our study, a reduction in WC was observed after three months of intervention, independent of intervention group or genotype. This may suggest that caloric restriction and increase in daily calorie consumption exert a more important influence on WC modification than IL6R genotype. As evidence suggests that adipocytes secrete different cytokines, which in turn may regulate adipose tissue size (Mohamed-Ali et al., 1997), the reduction in WC in study participants reflect a reduced adipose tissue size, with consequent lower cytokine production, lower insulin resistance, and lower fasting plasma glucose level. In fact, in our study a significant reduction in serum glucose levels was observed after intervention. When analyzing by genotype, individuals with the A allele exhibited higher levels at the beginning of the study (AA was the highest, $\mathrm{AC}$ intermediate, $\mathrm{CC}$ lowest), but showed a better response to intervention, with significant reduction in glucose levels.

For triglyceride levels, a better response to intervention was observed in individuals bearing allele A. As a significant reduction was observed for AA genotype individuals in the NI and NIE groups but in AC genotype only in NI, we can hypothesize that individuals with AC genotype need a combination of exercise and nutritional intervention for a better response. The literature presents conflicting results on the association of $48867 \mathrm{~A}>\mathrm{C}$ IL6R polymorphism with triglyceride levels, with no association observed (Hamid et al., 2005) or significantly elevated triglyceride levels for individuals with genotypes AA and AC, when compared to CC (Esteve et al., 2006).

The absence of significant change for HDL-C in any of our study groups is similar to the finding of a study conducted in elderly men and women after 12 months of physical activity intervention (Wang et al., 2012). Also, in a recent study with individuals submitted to a moderate intensity exercise training for 3 months on bicycle ergometers, no change in lowdensity lipoprotein cholesterol or HDL-C levels was observed but rather an increase in the antioxidative capacity of HDL subfractions, suggesting a dissociation between the quantitative and qualitative aspects of HDL after short-term exercise training (Casella-Filho et al., 2011).

Regarding blood pressure, a significant reduction in systolic blood pressure was observed in individuals bearing the A allele, suggesting a better response associated with A allele presence. No significant alteration in diastolic blood pressure was observed. Similarly, in the study of Esteve et al. (2006), no significant difference in systolic blood pressure or diastolic blood pressure was observed.

An overall analysis of the parameters studied before and after three months of lifestyle modification intervention showed improvement in WC and triglyceride and glucose levels, independently of intervention group or genotype. This suggests that once a nutritional intervention is adopted, with reduction in calorie intake, individuals with MetS would benefit. As mentioned previously, it is possible that the intensity or extent of exercise intervention performed in our study was not sufficient for an independent change in many MetS inclusion criteria. Recent studies have also reported a significant decrease in several clinical parameters in MetS patients after nutritional intervention, such as WC and triglycerides (Ren et al., 2007; 
Pimentel et al., 2010; Busnello et al., 2011).

The present analysis of the Asp358Ala (48867A>C) IL6R polymorphism supports that literature data on A allele as a possible genetic risk factor, since participants bearing this allele (genotypes AA and AC) showed at enrollment more severe conditions regarding MetS inclusion criteria. On the other hand, these individuals had a better response to lifestyle modification intervention compared to CC genotype participants, suggesting that nutritional control and exercise practice could prevent risks associated with MetS, such as cardiovascular disease and type 2 diabetes, more efficiently in this group of individuals. Further studies should be conducted to better explore this possible new role of allele $\mathrm{A}$ in the response to lifestyle modification intervention.

\section{ACKNOWLEDGMENTS}

Research supported in part by Conselho Nacional de Desenvolvimento Científico e Tecnológico (CNPq). The authors thank all the MERC team and the Molecular Biology Laboratory staff for contributing to the accomplishment of this study. Special credit goes to Dr. João Feliz Duarte de Moraes for statistical support.

\section{REFERENCES}

Akintunde AA, Ayodele OE, Akinwusi PO and Opadijo GO (2011). Metabolic syndrome: comparison of occurrence using three definitions in hypertensive patients. Clin. Med. Res. 9: 26-31.

Busnello FM, Bodanese LC, Pellanda LC and Santos ZE (2011). Nutritional intervention and the impact on adherence to treatment in patients with metabolic syndrome. Arq. Bras. Cardiol. 97: 217-224.

Casella-Filho A, Chagas AC, Maranhão RC, Trombetta IC, et al. (2011). Effect of exercise training on plasma levels and functional properties of high-density lipoprotein cholesterol in the metabolic syndrome. Am. J. Cardiol. 107: 1168-1172.

Cornier MA, Dabelea D, Hernandez TL, Lindstrom RC, et al. (2008). The metabolic syndrome. Endocr. Rev. 29: 777-822.

Dalle GR, Calugi S, Centis E, Marzocchi R, et al. (2010). Lifestyle modification in the management of the metabolic syndrome: achievements and challenges. Diabetes Metab. Syndr. Obes. 3: 373-385.

Das SK and Elbein SC (2006). The genetic basis of type 2 diabetes. Cellscience 2: 100-131.

De Luca C and Olefsky JM (2006). Stressed out about obesity and insulin resistance. Nat. Med. 12: 41-42.

Duvnjak L and Duvnjak M (2009). The metabolic syndrome - an ongoing story. J. Physiol. Pharmacol. 60 Suppl 7: 19-24.

Esteve E, Villuendas G, Mallolas J, Vendrell J, et al. (2006). Polymorphisms in the interleukin-6 receptor gene are associated with body mass index and with characteristics of the metabolic syndrome. Clin. Endocrinol. 65: 88-91.

Fernández-Real JM and Ricart W (2003). Insulin resistance and chronic cardiovascular inflammatory syndrome. Endocr. Rev. 24: 278-301.

Galicia JC, Tai H, Komatsu Y, Shimada Y, et al. (2004). Polymorphisms in the IL-6 receptor (IL-6R) gene: strong evidence that serum levels of soluble IL-6R are genetically influenced. Genes Immun. 5: 513-516.

Grundy SM, Cleeman JI, Daniels SR, Donato KA, et al. (2005). Diagnosis and management of the metabolic syndrome: an American Heart Association/National Heart, Lung, and Blood Institute Scientific Statement. Circulation 112: $2735-2752$.

Gupta A and Gupta V (2010). Metabolic syndrome: what are the risks for humans? Biosci. Trends 4: 204-212.

Hamid YH, Rose CS, Urhammer SA, Glumer C, et al. (2005). Variations of the interleukin-6 promoter are associated with features of the metabolic syndrome in Caucasian Danes. Diabetologia 48: 251-260.

Heinrich PC, Behrmann I, Haan S, Hermanns HM, et al. (2003). Principles of interleukin (IL)-6-type cytokine signalling and its regulation. Biochem. J. 374: 1-20.

IDF Clinical Guidelines Task Force (2006). Global guideline for type 2 diabetes: recommendations for standard, comprehensive, and minimal care. Diabet. Med. 23: 579-593.

Jones SA, Horiuchi S, Topley N, Yamamoto N, et al. (2001). The soluble interleukin 6 receptor: mechanisms of production and implications in disease. FASEB J. 15: 43-58.

Kim LH, Lee HS, Kim YJ, Jung JH, et al. (2003). Identification of novel SNPs in the interleukin 6 receptor gene (IL6R). 
Hum. Mutat. 21: 450-451.

Lahiri DK and Nurnberger JI Jr. (1991). A rapid non-enzymatic method for the preparation of HMW DNA from blood for RFLP studies. Nucleic Acids Res. 19: 5444.

Lakka HM, Laaksonen DE, Lakka TA, Niskanen LK, et al. (2002). The metabolic syndrome and total and cardiovascular disease mortality in middle-aged men. JAMA 288: 2709-2716.

Lazar MA (2006). The humoral side of insulin resistance. Nat. Med. 12: 43-44.

Meshkani R and Adeli K (2009). Hepatic insulin resistance, metabolic syndrome and cardiovascular disease. Clin. Biochem. 42: 1331-1346.

Mohamed-Ali V, Goodrick S, Rawesh A, Katz DR, et al. (1997). Subcutaneous adipose tissue releases interleukin-6, but not tumor necrosis factor-alpha, in vivo. J. Clin. Endocrinol. Metab. 82: 4196-4200.

Monteiro R and Azevedo I (2010). Chronic inflammation in obesity and the metabolic syndrome. Mediators. Inflamm. 2010.

Ordovas JM and Shen J (2008). Gene-environment interactions and susceptibility to metabolic syndrome and other chronic diseases. J. Periodontol. 79: 1508-1513.

Pimentel GD, Arimura ST, de Moura BM, Silva ME, et al. (2010). Short-term nutritional counseling reduces body mass index, waist circumference, triceps skinfold and triglycerides in women with metabolic syndrome. Diabetol. Metab. Syndr. 2: 13.

Qi L, Rifai N and Hu FB (2007). Interleukin-6 receptor gene variations, plasma interleukin-6 levels, and type 2 diabetes in U.S. Women. Diabetes 56: 3075-3081.

Rafiq S, Frayling TM, Murray A, Hurst A, et al. (2007). A common variant of the interleukin 6 receptor (IL-6r) gene increases IL-6r and IL-6 levels, without other inflammatory effects. Genes Immun. 8: 552-559.

Ren J, Zhu W, Dai H, Chen Z, et al. (2007). Nutritional intervention in the metabolic syndrome. Asia Pac. J. Clin. Nutr. (Suppl 16) 1: 418-421.

Santer FR, Malinowska K, Culig Z and Cavarretta IT (2010). Interleukin-6 trans-signalling differentially regulates proliferation, migration, adhesion and maspin expression in human prostate cancer cells. Endocr. Relat. Cancer 17: 241-253.

Sherry ST, Ward MH, Kholodov M, Baker J, et al. (2001). dbSNP: the NCBI database of genetic variation. Nucleic Acids Res. 29: 308-311.

Sociedade Brasileira de Cardiologia (2005). I Diretriz Brasileira de Diagnóstico e Tratamento da Síndrome Metabólica. Arq. Bras. Cardiol. 84 (Suppl I): 1-28.

Villareal DT, Miller BV 3rd, Banks M, Fontana L, et al. (2006). Effect of lifestyle intervention on metabolic coronary heart disease risk factors in obese older adults. Am. J. Clin. Nutr. 84: 1317-1323.

Wang H, Zhang Z, Chu W, Hale T, et al. (2005). Molecular screening and association analyses of the interleukin 6 receptor gene variants with type 2 diabetes, diabetic nephropathy, and insulin sensitivity. J. Clin. Endocrinol. Metab. 90: 1123-1129.

Wang X, Hsu FC, Isom S, Walkup MP, et al. (2012). Effects of a 12-month physical activity intervention on prevalence of metabolic syndrome in elderly men and women. J. Gerontol. A Biol. Sci. Med. Sci. 67: 417-424.

Wellen KE and Hotamisligil GS (2005). Inflammation, stress, and diabetes. J. Clin. Invest. 115: 1111-1119.

Wolford JK, Colligan PB, Gruber JD and Bogardus C (2003). Variants in the interleukin 6 receptor gene are associated with obesity in Pima Indians. Mol. Genet. Metab. 80: 338-343.

Yamada Y, Kato K, Hibino T, Yokoi K, et al. (2007). Prediction of genetic risk for metabolic syndrome. Atherosclerosis 191: 298-304.

Yu Z, Ye X, Wang J, Qi Q, et al. (2009). Associations of physical activity with inflammatory factors, adipocytokines, and metabolic syndrome in middle-aged and older chinese people. Circulation 119: 2969-2977. 